\title{
A tractable evolutionary model for the Minority Game with asymmetric payoffs
}

\author{
Pietro Dindo \\ CeNDEF, Department of Quantitative Economics, University of Amsterdam, Roetersstraat 11, \\ 1018 WB Amsterdam, the Netherlands
}

Received 31 October 2004; received in revised form 17 January 2005

Available online 2 May 2005

\begin{abstract}
We set up a simple behavioral model for a large population of agents who are repeatedly playing the Minority Game and whose interaction is modeled by means of the so-called replicator dynamics. This allows us to specify the dynamics of the aggregate variables, the number of agents choosing each side, in terms of a low-dimensional dynamical system that gives qualitatively the same results of the existing computational approaches. As an extension we introduce asymmetric payoffs, i.e., we analyze the case where the minority and majority payoffs are side dependent. In this case the fluctuations out of the equilibrium are qualitatively different. In particular, contrary to the previous case, they are associated with a difference in the average payoff gained by each side. Furthermore, a parameter region exists where the dynamics does not converge to any isolated periodic attractor.
\end{abstract}

(C) 2005 Elsevier B.V. All rights reserved.

PACS: 87.23.Ge; 89.65.Gh; 05.45.Ac

Keywords: Minority game; Nonlinear dynamical systems; Coordination and self-organization

\section{Introduction}

The Minority Game has been introduced as a stylized setting for modeling and analyzing the strategic interaction of a large number of agents. In the original

E-mail address: p.d.e.dindo@uva.nl. 
version of the game [1] an odd number of agents has to choose simultaneously whether to take side " 1 " or side " 0 ". Once the decisions are taken the agents who turn out to be in the minority side get a point, the others get nothing. The same game is repeated over time, the agents cannot communicate and they take their decisions on the basis of their common and symmetric information over the previous rounds. The fact that every winning strategy is not profitable anymore as soon as too many agents start using it, causes the competition to sit in the minority side never to settle down. The large literature [2] on the topic has primarily concentrated on the capability of the agents to self-organize and to maximize the total population payoff. This is compared to the benchmark case where the mixed strategy Nash equilibrium of the one stage game is played repeatedly. The two cases coincide for what concerns the mean of the fraction of agents taking each side and are usually distinguished only by looking at its variance. Most of the existing approaches are computational and the observed properties of the system are described by means of the tools of statistical mechanics and critical phenomena.

In order to tackle the same issue using a tractable model, we construct a dynamical system for the Minority Game and we analyze its behavior using local bifurcation analysis and by looking at the properties of the orbits generated by the system. At this first stage, in order to keep the structure of the model, as well as its mathematical formulation, as simple as possible, we consider agents using simple heuristics. For this case, in accordance with the literature, e.g. [3], we find that the variance of the fraction of agents taking each side remains strictly positive for large $N$. As an extension, we consider the case where the number of points awarded to a minority depends on the side where the minority is realized. This introduces an asymmetry in the system which causes the mixed strategy Nash equilibrium to differ from the level at which the system is maximizing the total population payoff. We apply our dynamical system to investigate the effect of the asymmetry in the payoffs on the coordination and self-organization properties of our agents. We are particularly concerned both with the distribution of payoffs among the population and with the total population payoff.

This paper is organized as follows: Section 2 presents the intuition behind our model and its mathematical formalization. Section 3 contains a qualitative analysis of the dynamics of our system and investigates the effect of the asymmetry on the population payoff. Section 4 concludes with a summary of the results and directions for future research.

\section{The model}

\subsection{Intuition}

The starting point is a population of agents who choose, period after period, to take either side " 0 " or side " 1 ". Our assumption is that the number of agents choosing each side is proportional both to how profitable that side has been so far and to the number of agents who were in that side the previous period. We could say, 
in this respect, that the number of agents taking a side is related to how popular that side is within the population. Where, with popular, we mean profitable during the previous periods and already chosen by other agents. Our agents have limited cognitive capabilities, in particular they do not consider the possibility that other agents may use the same "index of popularity" in taking their decisions. It is possible to think of more sophisticated agents who, for example, condition their choice on the past history or on the outcome of a set of predictors. In this case the corresponding dynamical system would be more complicated. At this stage of our investigation we want to keep the formulation of the model as simple as possible. We notice that despite its simplicity, our framework incorporates the main message of the Minority Game: a decision cannot be popular and winning at the same time. As long as a side is winning its popularity increases so that it is chosen by more and more agents, turning in the loosing side soon. The competition for choosing the minority side may never settle down.

\subsection{Formalization}

We call $x_{1, t}$ the fraction of agents that at time $t$ ends up in side " 1 " and $x_{0, t}=$ $1-x_{1, t}$ the fraction of agents in side " 0 " at time $t$. In principle every fraction is a number of the type $k / N$ with $k \in\{0, \ldots, N\}$ but, in practice, we use $x_{i, t} \in[0,1]$, $i=0,1$, which corresponds to considering a very large population of agents. In the original definition of the game, every agent who sits in a minority gets a payoff $\pi_{s}$ (minority $=$ success) and every agent in a majority gets $\pi_{f}<\pi_{s}$ (majority $=$ failure). Here, at time $t$, we consider:

$$
\pi_{i, t}=\pi\left(x_{i, t}\right)=\frac{\left(\pi_{s}+\pi_{f}\right)}{2}-\frac{\left(\pi_{s}-\pi_{f}\right)}{2} \tanh \left(\gamma\left(x_{i, t}-0.5\right)\right), \quad i=0,1 .
$$

Note that the limit as $\gamma \rightarrow \infty$ gives back the original minority rule which pays $\pi_{i}=\pi_{s}$ if and only if $x_{i}<0.5$. If $\gamma$ is finite, the size of the minority also matters so that a smaller minority is rewarded with a higher payoff. As a difference with the original case, where $N$ is odd and finite, we consider an arbitrarily large value of $N$ so that, in general, the point $x_{i}=0.5$ is also in the domain of the payoff function given in Eq. (1). A generalization of the payoff function allows us to investigate the case where an asymmetry is introduced in the payoff structure. We are particularly interested in the case where one side, say side " 0 ", is less risky than the other: being in the minority is rewarded with less points but ending up in the majority causes a lower loss. This idea is realized by taking the values of the majority and minority payoffs as side dependent. We introduce this asymmetry by means of the parameter $\alpha \in[0,1]$ in the definition of $\pi_{0, t}$ :

$$
\pi_{0, t}=\pi_{\alpha}\left(x_{0, t}\right)=\frac{\left(\pi_{s}+\pi_{f}\right)}{2}-(1-\alpha) \frac{\left(\pi_{s}-\pi_{f}\right)}{2} \tanh \left(\gamma\left(x_{0, t}-0.5\right)\right) .
$$

If $\alpha=0$ we are back in the symmetric case. When $\alpha \neq 0$ side " 0 " is less "risky" than side " 1 ". By that we mean that the difference between the highest and the lowest 
payoff is nearly ${ }^{1}(1-\alpha)\left(\pi_{s}-\pi_{f}\right)$ on side " 0 " and nearly $\left(\pi_{s}-\pi_{f}\right)$, thus higher, on side " 1 ". When $\alpha=1$ we have $\pi_{0, t}=\left(\pi_{s}+\pi_{f}\right) / 2, \forall t$. Taking side " 0 " has no strategic uncertainty anymore. ${ }^{2}$

In accordance with Section 2.1 we assume that, at period $t+1$, the number of agents on a side is proportional both to the points that side has earned and to how many agent were on that side at period $t$. The replicator dynamics [5] implements this mechanism. It is commonly used to model the interaction of agents in evolutionary games [6] and, according to it, the fraction $x_{i, t+1}$ is given by

$$
x_{i, t+1}=\frac{x_{i, t} u_{i, t}}{x_{1, t} u_{1, t}+x_{0, t} u_{0, t}},
$$

where $u_{i, t}$ measures the points that side $i$ has earned and it is given by the discounted sum, with coefficient $\mu$, of the past gained payoffs:

$$
u_{i, t}=\mu u_{i, t-1}+(1-\mu) \pi_{i, t} .
$$

We have now all the elements to write down a dynamical system for the Minority Game. We note that $x_{0, t}=1-x_{1, t}$, so that the system is three-dimensional and given by

$$
\begin{aligned}
& x_{1, t+1}=\frac{x_{1, t} u_{1, t}}{x_{1, t} u_{1, t}+\left(1-x_{1, t}\right) u_{0, t}}, \\
& u_{1, t+1}=\mu u_{1, t}+(1-\mu) \pi\left(x_{1, t+1}\right), \\
& u_{0, t+1}=\mu u_{0, t}+(1-\mu) \pi_{\alpha}\left(1-x_{1, t+1}\right) .
\end{aligned}
$$

We characterize the dynamics for different values of the parameters in the following section. We are particularly interested in behavior of the distribution of payoffs among the population as a function of the degree of asymmetry $\alpha$.

\section{Some results}

Here we briefly present the qualitative analysis of the dynamics of the model we have set up in the previous section. We treat as different cases the situation where $\alpha=0$ (symmetric case) and the situation were $\alpha \neq 0$ (asymmetric case). In both cases, $x_{1}=0.5$ is the fixed point of the system as well as the unique symmetric mixed strategy Nash equilibrium. ${ }^{3}$ An insight about the difference between the two cases comes from the comparison of the average population payoff, $\bar{\pi}=x_{1} \pi_{1}+x_{0} \pi_{0}$, as a function of $x_{1}$ (see Fig. 1). In the symmetric case, $x_{1}=0.5$ is both the (unique symmetric mixed strategy) Nash equilibrium and the optimal level which maximizes

\footnotetext{
${ }^{1}$ Note that this difference depends on $\gamma$. The result holds with equality only when $\gamma \rightarrow \infty$.

${ }^{2}$ This situation resembles the setting of the El Farol problem [4] when going to the bar is risky (taking side " 1 ") while staying at home (taking side " 0 ") gives always the same payoff.

${ }^{3} X_{1}=N x_{1}=N / 2$ is in fact the expected value of a binomial random variable with parameters $N$ and $p=0.5$. The latter can be shown to be the symmetric mixed strategy Nash equilibrium of the Minority Game for all $N$ and for all $\alpha$.
} 

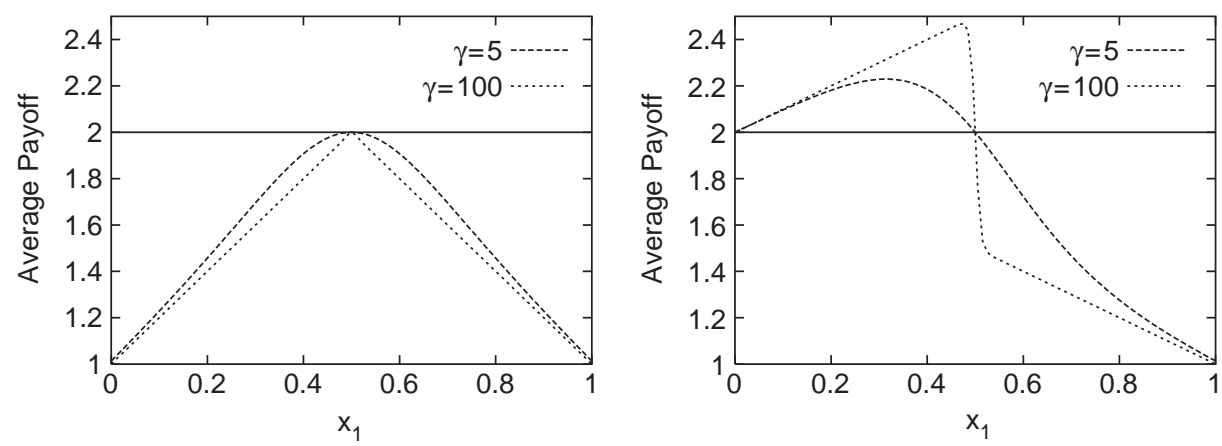

Fig. 1. Average population payoff, $\bar{\pi}$, is plotted as function of $x_{1}$, for two different values of $\alpha$. On the left for $\alpha=0$, on the right for $\alpha=1$. Both plots illustrate both the case of $\gamma=5$ as well as the case of $\gamma=100$. The values of the payoffs are $\pi_{s}=3$ and $\pi_{f}=1$.

the average payoff of the population so that, $\bar{\pi}(0.5)=\bar{\pi}_{M A X}$. As a result, when the system is not stable at the mixed strategy Nash equilibrium, its fluctuations considerably lower the average population payoff. In the asymmetric case, $x_{1}=0.5$ is still the (unique symmetric mixed strategy) Nash equilibrium but $\bar{\pi}(0.5) \neq \bar{\pi}_{M A X}$. In this second case fluctuations around the mixed strategy Nash equilibrium may lead to a higher average population payoff then $\bar{\pi}(0.5)$. We investigate this circumstance in what follows.

\subsection{The symmetric case}

When $\alpha=0$ the system (5) has three different fixed points: $x_{1}=0,1$ and 0.5 . As already noted, the latest corresponds to the unique mixed strategy Nash equilibrium of the game. It can be shown that the two first fixed points are always unstable. The stability of $x_{1}=0.5$ primarily depends on $\mu$ and $\gamma$. When $\mu=0$ the system is onedimensional and the stability condition for $x_{1}=0.5$ in terms of $\gamma$ is given by ${ }^{4}$

$$
\gamma<4 \frac{\pi_{s}+\pi_{f}}{\pi_{s}-\pi_{f}}
$$

The previous condition means that the system loses stability as soon as the payoff function is steep enough at the fixed point. In the case $\mu \neq 0$ it can be shown that the system is stable in a bigger parameter region but a similar condition still holds. In general the steady state turns into a two-cycle by means of a period doubling bifurcation. The dynamics in the instability region depends again on the value of $\mu$. When $\mu=0$ the system has different coexisting two-cycles, an infinite number as $\gamma \rightarrow \infty$. When $\mu \neq 0$, the system shows coexistence of stable two-cycles and stable four-cycles. For a given initial condition, the system converges to either one of the two-cycles or one of the four-cycles. We illustrate this feature in the first panel of

\footnotetext{
${ }^{4}$ The system depends also on $\pi_{s} / \pi_{f}$. If we normalize $\pi_{f}$ to one, the system does not show qualitative changes for other values of $\pi_{s}$.
} 

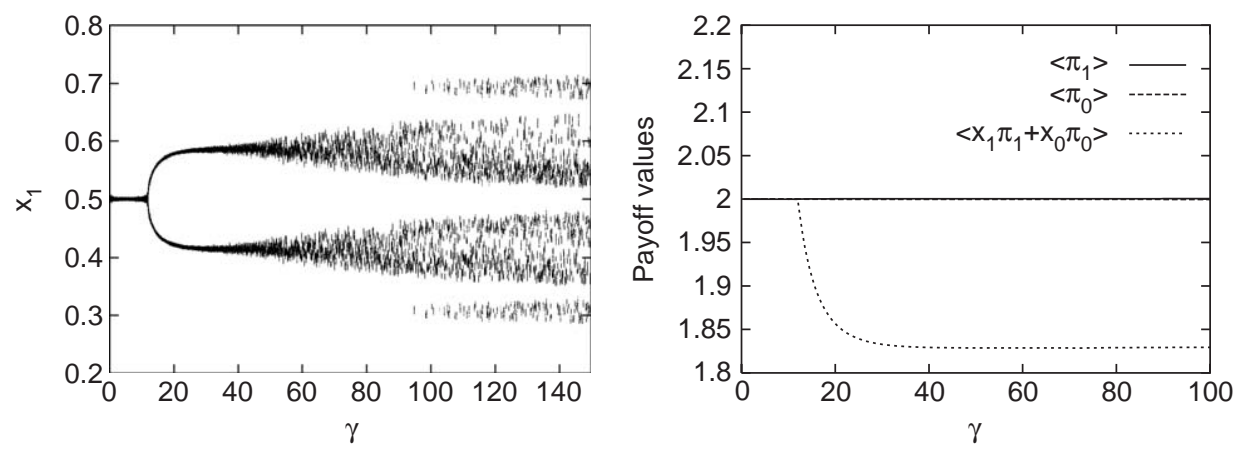

Fig. 2. Left panel shows a bifurcation diagram w.r.t. $\gamma$. For every value of $\gamma$ after a transient of 900 iterations an orbit of 100 points is plotted. When we add some noise $\left(z_{t}\right.$ i.i.d., $\left.\sim N(0,0.001)\right)$ to $x_{1}$ the system jumps between different two-cycles and four-cycles as soon as $\gamma$ is high enough. Note that the noise does not significantly affect the system when $\gamma$ is low. Right panel shows the time average (1000 periods) of $\bar{\pi}, \pi_{1}$ and $\pi_{0}$ as a function of $\gamma$. When $\gamma$ is such that the system loses stability, $\langle\bar{\pi}\rangle$ decreases while $\left\langle\pi_{1}\right\rangle$ and $\left\langle\pi_{0}\right\rangle$ are always equal. In both plots $\pi_{s}=3, \pi_{f}=1, \mu=0.2$ and $\alpha=0$.

Fig. 2, where we show, for a range of values of $\gamma$, how the addition of a little noise causes the orbits to jump among the different coexisting attractors.

It is interesting to characterize the stability and instability region also in terms of time average payoffs. Let \langle\rangle denote the time average along an orbit. In the stability region $\pi_{1}=\pi_{0}$ and $x_{1}=0.5$ so that $\bar{\pi}$ achieves a maximum. When fluctuations are present the symmetry of the system implies $\left\langle x_{1}\right\rangle=0.5$ and $\left\langle\pi_{1}\right\rangle=\left\langle\pi_{0}\right\rangle=\bar{\pi}_{M A X}$ but now $\langle\bar{\pi}\rangle$ is lower than $\bar{\pi}_{M A X}$. The fluctuations have no effect on average individual payoff but a negative effect in terms of average population payoff. The reason for that is clear from Fig. 1: out of the equilibrium the value of $\bar{\pi}$ is always lower than $\bar{\pi}(0.5)$. The right panel of Fig. 2 shows how time average payoffs change with $\gamma$.

\subsection{The asymmetric case}

When $\alpha \neq 0$ the system (5) has the same three fixed points as in the symmetric case. The only one of a certain interest is, again, $x_{1}=0.5$ which corresponds to the unique symmetric mixed strategy equilibrium of the underlying game. Its stability condition in terms of $\gamma$ (when $\mu=0$ ) is given by

$$
\gamma<\frac{8}{2-\alpha} \frac{\pi_{s}+\pi_{f}}{\pi_{s}-\pi_{f}} .
$$

Note that the previous condition coincides with (6) when $\alpha=0$ but, when $\alpha \neq 0$, the system is stable for $\gamma$ in a larger interval, if we keep $\pi_{s}$ and $\pi_{f}$ fixed. As before it can be shown that $\forall \mu$ the fixed point $x_{1}=0.5$ becomes unstable by means of a period doubling bifurcation but the total bifurcation structure of the system is now richer. In particular, values of the parameters exist where the dynamics produces nonperiodic orbits. Furthermore, when the system is one-dimensional $(\mu=0)$ the 

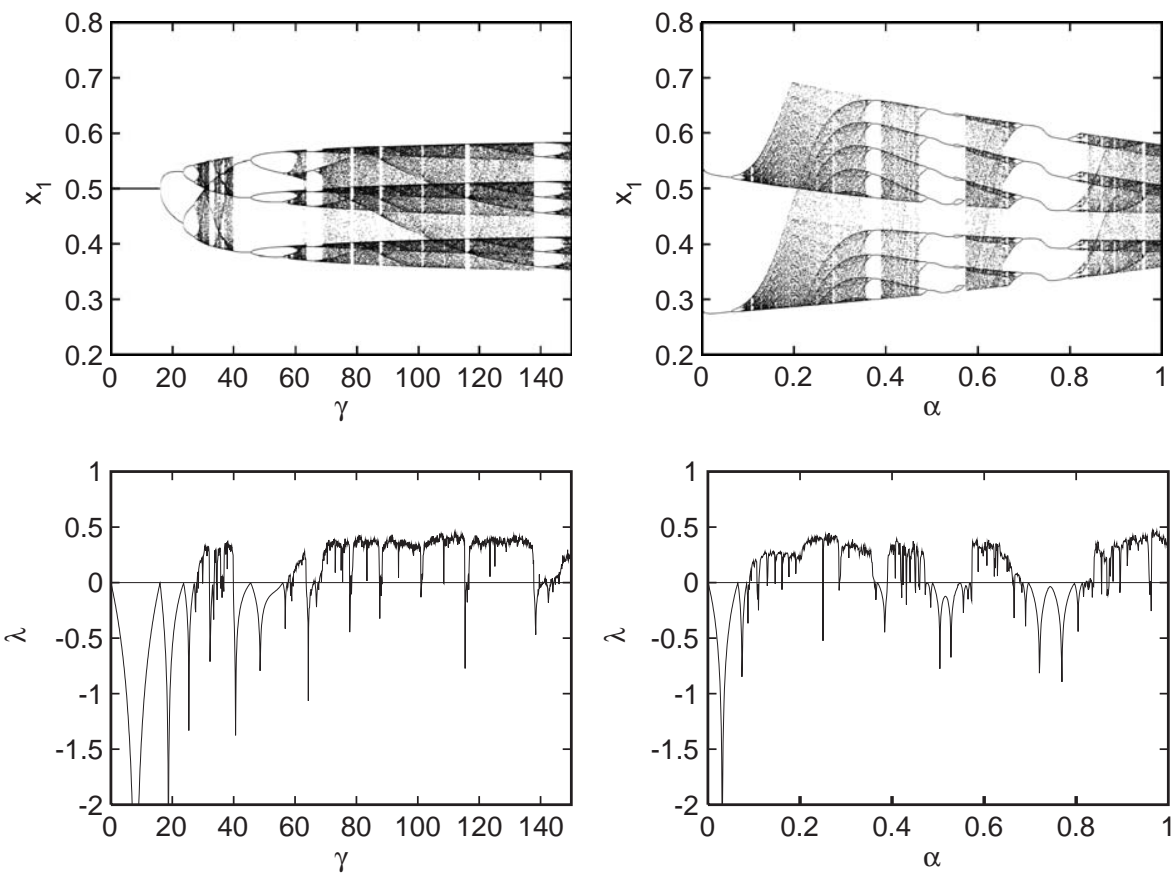

Fig. 3. On the top panels two bifurcation diagrams are shown. In both cases after a transient of 900 iterations, orbits of 100 points are plotted. On the right $\gamma \in[0,150]$ and $\alpha=1$ while on the left $\alpha \in[0,1]$ and $\gamma=100$. On the bottom panel Lyapunov exponents are shown for the same ranges of the parameters. Note that the value of $\gamma$ and $\alpha$ where a bifurcation occurs corresponds to $\lambda=0$ and that when $\lambda>0$ the dynamics does not settle down to any period attractor. The value of the payoffs are fixed to $\pi_{s}=3$ and $\pi_{f}=1$. In all the cases $\mu=0$.

presence of a three-cycle implies topological chaos [7]. Fig. 3 presents, in the case ${ }^{5}$ of $\mu=0$, a bifurcation diagram and Lyapunov exponents both as a function of $\gamma$ for $\alpha=1$ and as a function of $\alpha$ for $\gamma=100$. In the first bifurcation diagram, as $\gamma$ increases, the period doubling bifurcation is followed by a cascade of bifurcation of the same type and by a tangent bifurcation which creates a three-cycle $(\gamma \sim 40)$. When $\gamma$ is higher a region with positive Lyapunov exponents is present. In the second bifurcation diagram, the system converges to a two-cycle only when $\alpha$ is lower then 0.1 . As soon as a higher degree of asymmetry is introduced, the system exhibits positive Lyapunov exponents.

We turn to the analysis of time average payoffs. In the stability region $\pi_{1}=\pi_{0}=\bar{\pi}$ holds but, contrary to the symmetric case, now $\bar{\pi} \neq \bar{\pi}_{M A X}$ (see also Fig. 1). In the instability region the dynamics is not symmetric anymore so that the aggregate attendance is no longer distributed around the mixed strategy Nash equilibrium level. Furthermore the fluctuations are not exclusively associated with a lower

\footnotetext{
${ }^{5}$ The case of $\mu \neq 0$ is a smoothed version of the one with no memory.
} 

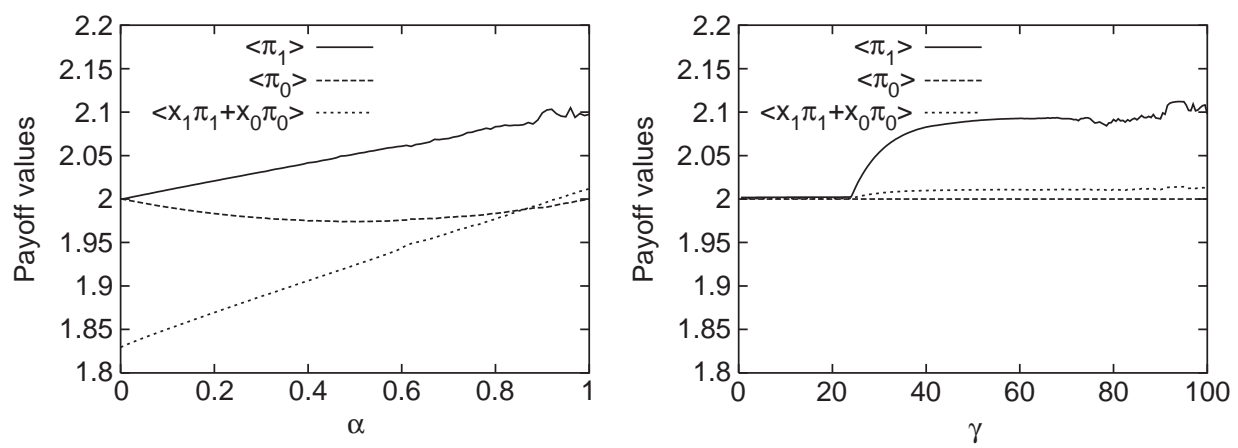

Fig. 4. Right panel shows the 1000 periods time average of $\bar{\pi}, \pi_{1}$ and $\pi_{0}$ as a function of $\gamma$ for $\alpha=1$. On the left, the same time average payoffs as a function of $\alpha$ for $\gamma=100$. In all the plots $\pi_{s}=3, \pi_{f}=1$ and $\mu=0.2$.

average population payoff as before. In particular, $\langle\bar{\pi}\rangle$ increases with $\alpha$ and $\left\langle\pi_{1}\right\rangle$ is now always higher than $\left\langle\pi_{0}\right\rangle$ (for both effects see the left panel of Fig. 4). The side associated with a higher "risk", i.e., where the difference between the majority and the minority payoff is larger, has a higher time average payoff. We present the behavior of the time average payoffs as a function of the bifurcation parameter $\gamma$ in the right panel of Fig. 4 which should be compared with the right panel of Fig. 2.

\section{Conclusions}

We have set up a tractable low-dimensional dynamical system that replicates the interaction of a large number of agents who are repeatedly playing the Minority Game. In the symmetric region, we have qualitatively obtained the same results as known in the literature in the case of agents using simple heuristics: the fractions of agents are distributed around the level which corresponds to the mixed strategy Nash equilibrium and the out-of-equilibrium oscillations considerably lower the average population payoff for a large value of $\gamma$. We have extended the problem by considering the asymmetric case where side "0" and side "1" have different associated "risks". In this case a parameter region exists where the dynamics does not converge to any isolated periodic cycle. In particular, contrary to the previous case, the mixed strategy Nash equilibrium does not seem to offer a good approximation to the out-of-equilibrium aggregate dynamics. Furthermore the payoff associated with the more risky side is higher than the other and the fluctuations are possibly associated with an increase in the average population payoff.

In order to draw more general conclusions we need to set up a similar dynamical system where more sophisticated agents are interacting. This, together with a precise characterization of the dynamics for more values of the parameters, will be the content of our future research. 


\section{Acknowledgements}

I would like to express my gratitude to Cees Diks and Jan Tuinstra for helpful discussions. The usual disclaimer applies.

\section{References}

[1] D. Challet, Y.-C. Zhang, Emergence of cooperation and organization in an evolutionary game, Physica A 246 (1997) 407.

[2] For a large collection of papers concerning both the analytical and numerical explorations of the minority game see http://www.unifr.ch.econophysics/.

[3] N.F. Johnson, et al., Crowds effects and volatility in a competitive market, Physica A 269 (1999) 1.

[4] W.B. Arthur, Inductive reasoning and bounded rationality, Am. Econ. Assoc. 84 (1994) 406.

[5] P. Taylor, L. Jonker, Evolutionary stable strategies and game dynamics, Math. Biosci. 40 (1978) 145.

[6] J.W. Weibull, Evolutionary Game Theory, MIT Press, Cambridge, MA, 1995.

[7] T.-Y. Lie, J.A. Yorke, Period three implies chaos, Am. Math. Monthly 82 (1975) 985. 